\title{
Modeling of Quench Limit for Steady State Heat Deposits in LHC Magnets
}

\author{
Dariusz Bocian, Bernd Dehning, and Andrzej Siemko
}

\begin{abstract}
A quench, the transition of a conductor from the superconducting to the normal conducting state, occurs irreversibly in the accelerator magnets if one of the three parameters: temperature, magnetic field or current density exceeds a critical value. Energy deposited in the superconductor by the particle beams provokes quenches detrimental for the accelerator operation. In particular if particles impacting on the vacuum chamber and their secondary showers depose energy in the magnet coils. The Large Hadron Collider (LHC) nominal beam intensity is $3.2 \cdot 10^{14}$ protons. A quench occurs if a fraction of the order of $10^{7}$ protons per second is lost locally.

A network model is used to simulate the thermodynamic behavior of the magnets. The heat flow in the network model was validated with measurements performed in the CERN magnet test facility. A steady state heat flow was introduced in the coil by using the quench heaters implemented in the LHC magnets. The value of the heat source current is determined by the network model and the magnet coil current which is required to quench the coil is predicted accordantly. The measured and predicted value comparison is regarded as a sensitive test of the method.
\end{abstract}

Index Terms-Heat flow model, LHC superconducting magnets, quench level calculations, steady state heat deposits.

\section{INTRODUCTION}

$\mathbf{T}$ HE high beam intensities in the LHC [1] require a careful control of the beam losses around the LHC ring. The beam losses, involving significant numbers of particles, may have severe consequences for the accelerator equipment [2]. Several systems are designed to ensure safe operation of the LHC, such as beam dumps, collimators and beam absorbers, the beam loss monitors and the magnet quench protection [3]. The loss durations for particle losses range from a few ns (one bunch) to several seconds, depending on the specific failure mode [4]. Protection from multi-turn beam failures relies mainly on the fast monitors that detect a beam losses early enough to issue a beam dump request immediately if the loss exceed the acceptable level. Steady state losses are mainly caused by the debris of the proton-proton interactions at experimental insertions and by the residual beam gas interactions. The dominant loss locations are the interaction and collimation regions and the nearby dispersion suppressors [2]. The continuous energy deposition in the main LHC magnets due to the beam losses implies a continuous evacuation of the heat from the coil to keep the temperature in the superconducting cables below the critical temperature.

Manuscript received August 28, 2007.

D. Bocian is with CERN, Geneva, Switzerland and also with IFJ PAN Krakow, Poland (e-mail: Dariusz.Bocian@cern.ch; Dariusz.Bocian@ifj.edu.pl).

B. Dehning and A. Siemko are with CERN, Geneva, Switzerland.

Color versions of one or more of the figures in this paper are available online at http://ieeexplore.iee.org.

Digital Object Identifier 10.1109/TASC.2008.921338
The heat flow at steady state is mainly limited by the size of the helium channels and the heat conduction of cable insulation. The power dissipation in the superconducting magnet components leads to a complex process of the heat flow, but in many cases a simplified model for heat transfer is sufficient. In this paper the Network Models developed for the main LHC superconducting magnets are presented. The Section II is devoted to the Network Model construction and the way that superconducting cable structure is transformed into a thermal resistance network. In the Section III the basic assumptions of the simulations are discussed. For the energy deposition by a beam loss an expected profile of the heat deposition is used resulting in a heat flow-temperature diagram of the coil of a magnet. The model validation with the measurements is the subject of the Section IV. The experimental setup is discussed and the results of the simulations and measurements and their relative differences for the study case of one magnet family are shown in this section. The detailed results of the measurements and details of the experimental setups are presented in [5], [6]. The quench limits of two families of the main LHC magnets operating at $4.5 \mathrm{~K}$, for a Gaussian beam loss profile are also presented.

In this paper only steady-state conditions are considered, the transient conditions are the subject of the other paper presented to this conference [7].

\section{NETWORK MODELS}

In the proposed modeling method, the four families of the main LHC magnets are considered (cf. Fig. 1). Namely the main bending dipole $\mathrm{MB}$ operating at $1.9 \mathrm{~K}$, main focusing quadrupole MQ operating at $1.9 \mathrm{~K}$, as well as the quadrupole magnets used in the dispersion suppressors; MQM (Insertion Region Quadrupole) operating at $1.9 \mathrm{~K}$ and long straight sections MQM and MQY (Insertion Region Wide Aperture Quadrupole) operating at $4.5 \mathrm{~K}$. In this paper, as a first step, only the magnets cooled by normal liquid helium at $4.5 \mathrm{~K}$ are discussed. The case of magnets operating at $1.9 \mathrm{~K}$ will be available elsewhere [8].

The network models are developed to study the thermodynamic behavior of the magnet coils and to calculate the quench levels of the main LHC magnets for typical beam loss profiles. Fig. 2 shows schematically the construction of the 2-dimensional network model. The vital input information for the models is taken from technical drawings, which provide the details for creation of the net of thermal elements. Other required information is: the material properties e.g., heat conductivity, magnetic field distribution, temperature margin calculation, beam loss profiles and the model validation data. The other non beam loss sources of the heat loads to the magnet coil, like the eddy currents and magnetic hysteresis losses were also evaluated. The impact on the quench level from these sources was 


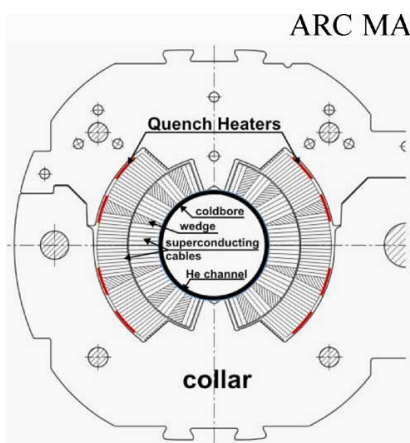

Main dipole, MB

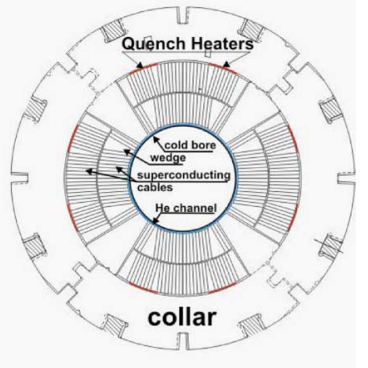

Main quadrupole, MQ

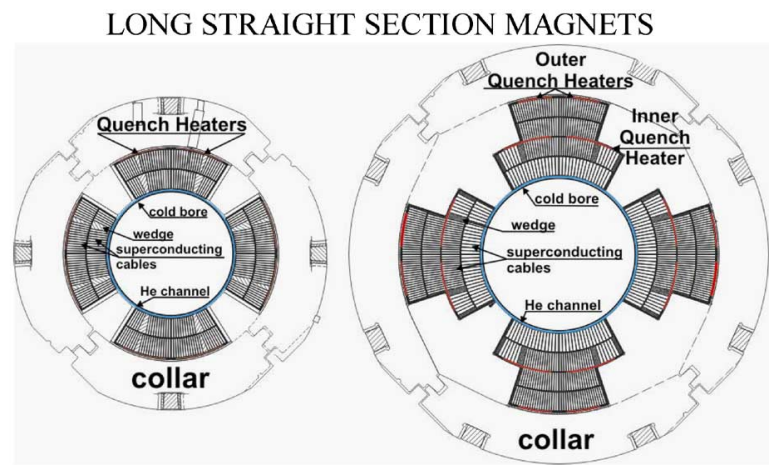

DS and LSS magnet, MQM

LSS magnet, MQY

Fig. 1. The cross section of the LHC main magnets. The magnets are operating at $1.9 \mathrm{~K}$ (MB, MQ and MQM) and 4.5K (MQM and MQY). DS means dispersion suppressor and LSS-Long Straight Section.

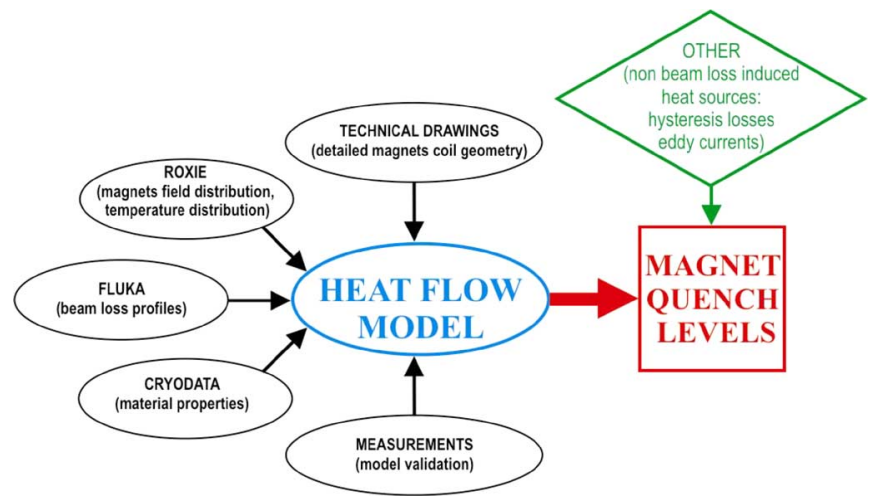

Fig. 2. Network Model construction overview.

estimated to be of the order of 1-2\% [9] and will be neglected in this study.

A picture and schematic cut view of a superconducting cable cross section is shown in Fig. 3. The strands are made of $\mathrm{Nb}$ - Ti filaments embedded in a copper matrix. The cable is wrapped with three layers of the polyimide insulation. The helium occupies the space between the wires. Detailed characteristics of the cable and insulation are presented in [8].

The detailed network model of a superconducting cable is shown in Fig. 4. The cable segmentation corresponds to its strands structure. In case of the metal part of the cable the thermal resistance element represents one strand. The same segmentation was applied to the insulation and helium channels. The cable sample was normalized to 1 meter of cable
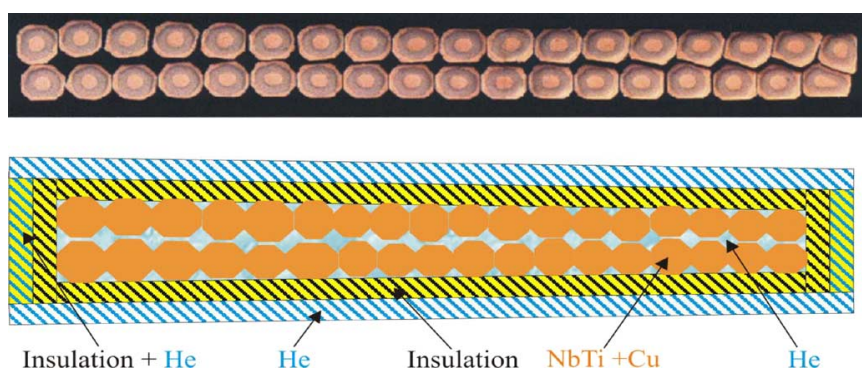

Fig. 3. Superconducting cable photo and schematic cut view. Insulation $+\mathrm{He}$ denotes the third layer of the insulation consisting of $18 \%$ of helium in $\mu$-channels and $82 \%$ of polyimide insulation.

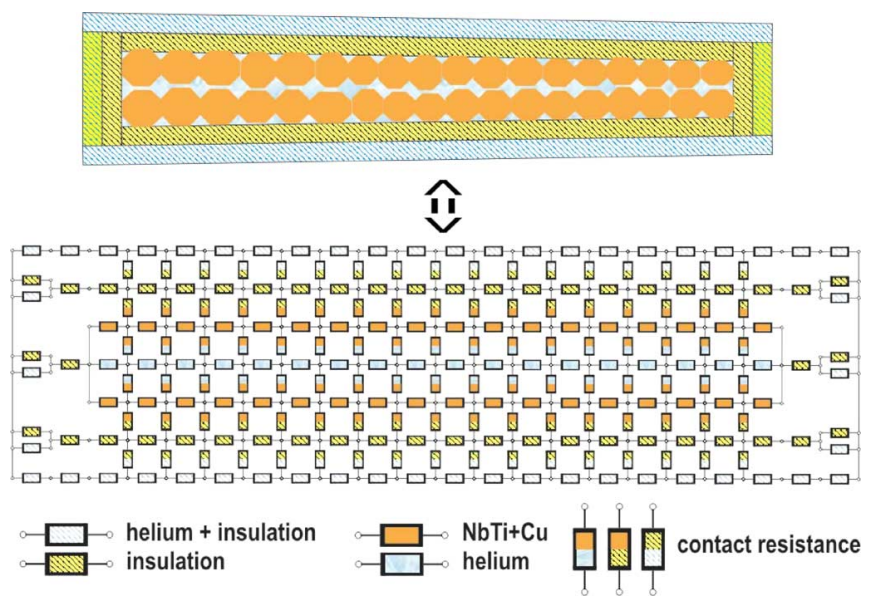

Fig. 4. The physical model of superconducting cable and its network model equivalent.

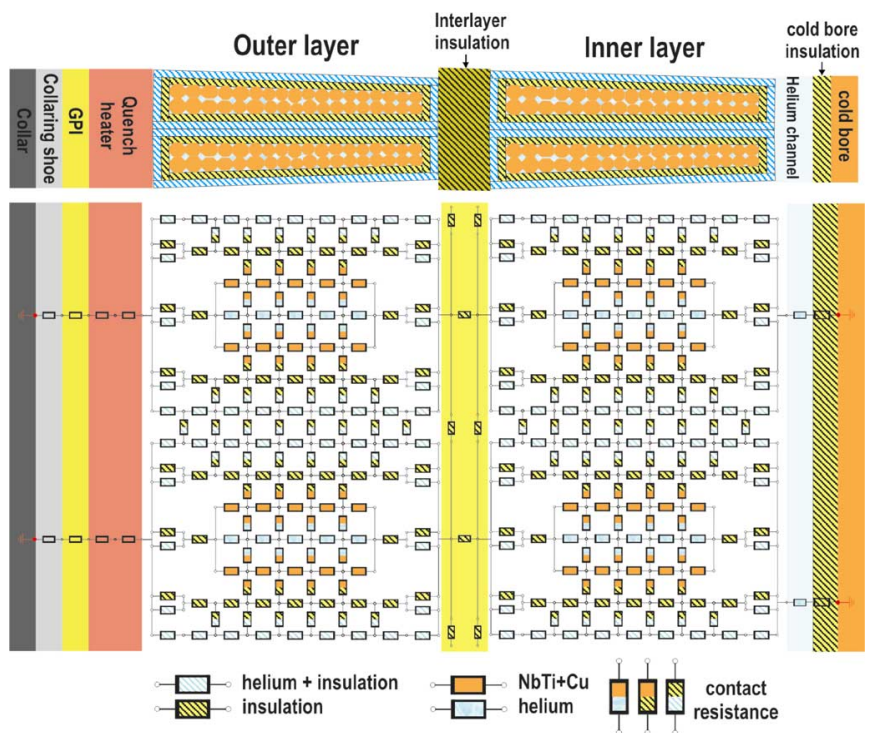

Fig. 5. Characteristic part of the superconducting coil network model.

length. The values of the thermal conductivity for the calculation of thermal resistance for each of the thermal resistors in the network model are taken from commercially available database [10] and literature [11]-[13].

As it is shown in Fig. 5, the magnet coil is modeled using the individual model of a cable as a building block. The thermal 


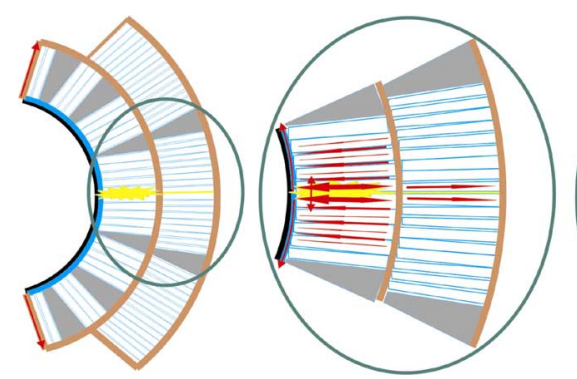

a)

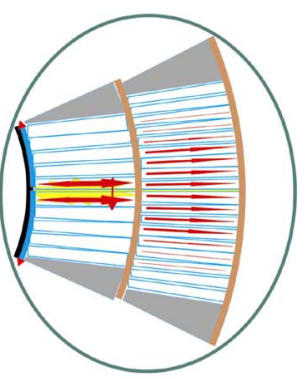

b)
Fig. 6. The heat flow scheme in the MB magnet at nominal operation (a) and at quench limit (b). Heat is evacuated from coil through helium channel around the cold bore in the ideal case. Arrows indicate the heat flow path through magnet.

resistances between the cables and between the cables and surrounding magnet structural elements, like the quench heaters, collars and the cold bore are implemented to complete the network model of the regular part of the magnet coil.

\section{NUMERICAL SimULATIONS}

The MB magnet cooled with liquid helium at $4.5 \mathrm{~K}$ was chosen as a study case for the heat flow in the superconducting coil at quench level. One can distinguish six characteristic heat flow mechanism in helium, which are relevant for this work. Namely: the superfluid helium heat conductivity, the nucleate boiling in the normal liquid helium, the convection and the heat conductivity of normal liquid helium, the convection and heat conductivity of the gaseous helium.

In the network model presented in Fig. 5 the volumes occupied by helium in the magnet coil are considered as the narrow channels. Moreover these volumes are considered as semi-closed volumes, without "easy" direct link to the helium bath in the magnet cold mass. The steady state beam loss heat load causes heating up the helium in the narrow channels to temperatures well above the helium critical temperature, which is reached already below the calculated quench limit. This results in creation of helium gas in the channels. The gaseous phase inside the narrow channels is described in the network model by a constant heat transfer coefficient, which is of the order of $70 \mathrm{~W} / \mathrm{m}^{2} / \mathrm{K}$ as extrapolated from [13]. In the case of a constant heat deposition at the quench level, the nucleate boiling and the convection heat transport mechanism inside the cable can be neglected due to creation of helium gas in these channels. Another property of the magnet, important for the heat flow in the magnet, is related to the ground insulation extending into the cold bore helium channel. The ground insulation edges ("flaps") are touching the cold bore insulation and are considered responsible for azimuthal segmentation of the channel around the cold bore [8]. This effect reduces significantly convective heat flow in a region already critical with respect to heat extraction.

The two main paths of the heat evacuation have been identified in relation to the helium temperature distribution in the channel around the cold bore (Fig. 6). The arrows indicate the main heat flow path at nominal operation (left) and at quench limit (right).

Fig. 7 shows the results of the case study for the main dipole magnet immersed in helium bath at $4.5 \mathrm{~K}$ at the quench level.

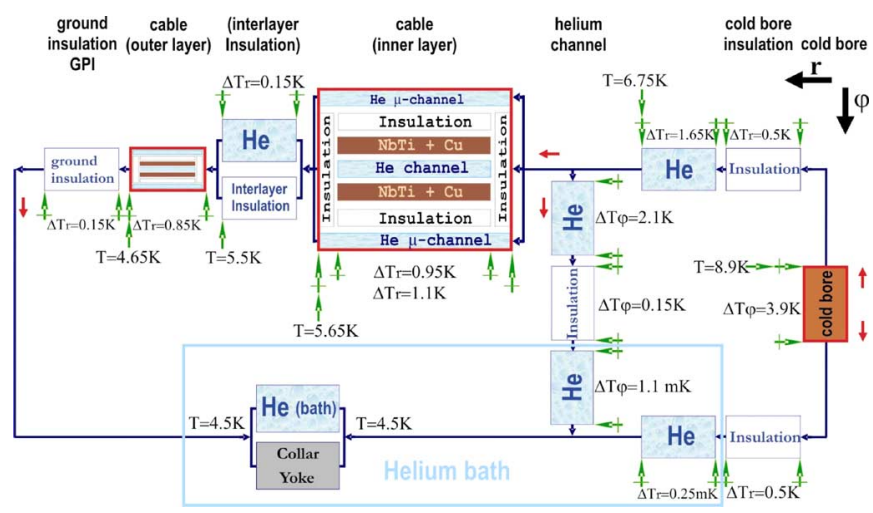

Fig. 7. Study case of heat flow in the magnet coil at quench for an energy deposition distribution shown in Fig. 8.
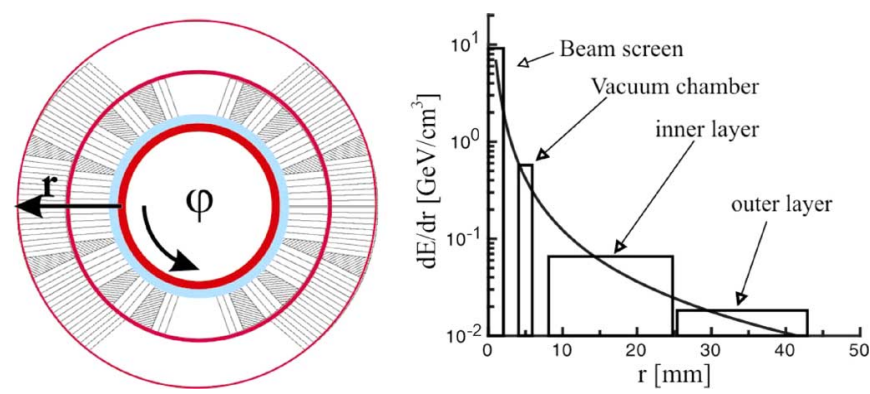

Fig. 8. The schematic of the heat deposits in the magnet coil (left) and the radial energy density along the most exposed azimuth [14].

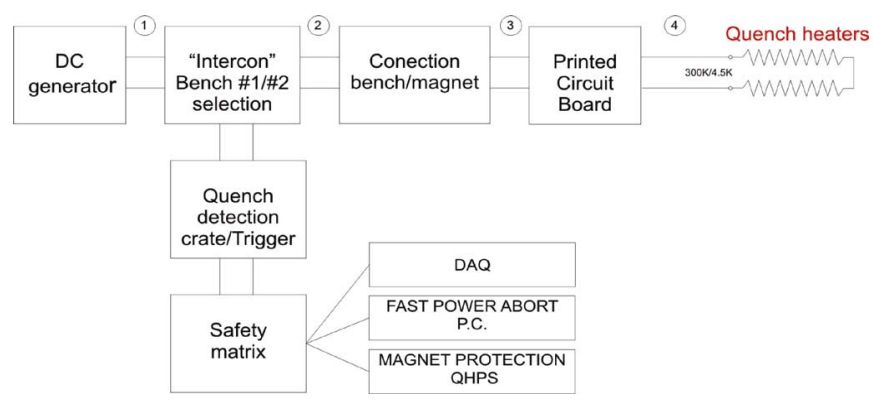

Fig. 9. A sketch of experimental setup for quench heater provoked quenches. The sketch shows the quench heaters connection during tests.1-cabling between the quench heater patch panel and "intercon", 2-cabling between racks and test bench, 3-instrumentation cable of the magnet, 4-capillary cable $300 \mathrm{~K} / 4.5 \mathrm{~K}$.

The heat deposition profile of the beam loss is shown in Fig. 8 [14]. The absolute and relative temperatures in the radial and azimuthal directions are indicated in the Fig. 7. As one can expect, for the heat flow barriers such as the cable insulation and the ground insulation in the cold bore channel, significant temperature drops are calculated.

\section{MOdel VALidation AND Simulation RESUlts}

The existing, built-in quench heaters in the main LHC magnets are very favorable for the studies devoted to the quench limit estimation. The experimental setup, which consists of built-in quench heaters and DC power supply, allows provoking quenches at different levels of the magnet current. A sketch of experimental setup is presented in Fig. 9. The quench heaters 


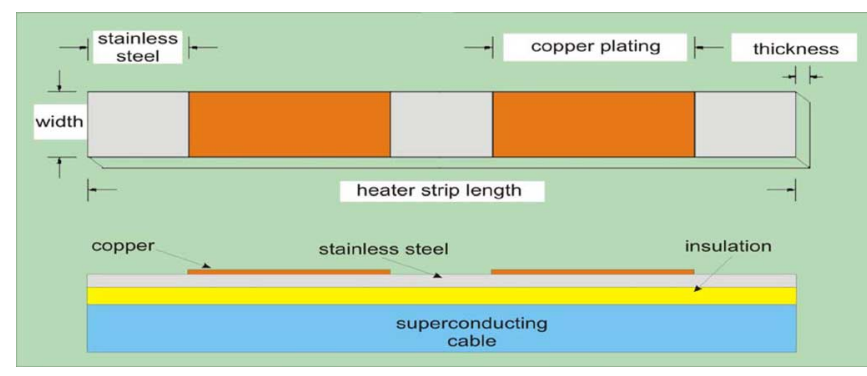

Fig. 10. The quench heater strip with the copper plated area for the LHC magnets.

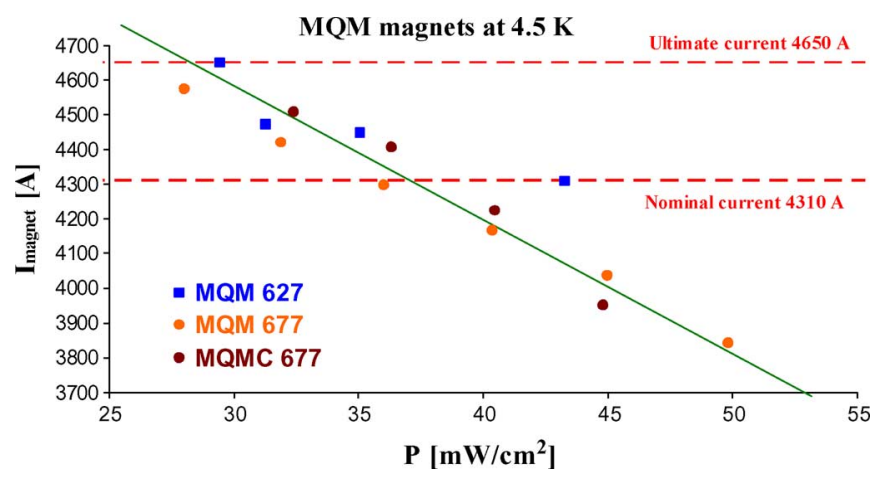

Fig. 11. Result of the measurements with a dedicated experimental setup (markers) and simulation (solid line) of one of the magnet family, MQM. A dedicated experimental setup consists of built-in quench heaters and DC power supply. The power dissipated in the quench heaters is shown on $\mathrm{x}$ axis and corresponding magnet quench current is depicted on y-axis.

installed in the LHC magnets are made of $25 \mu \mathrm{m}$ thick ${ }^{1}$ stainless steel strips. The strips are positioned along the magnet between the coil and the collars (see Fig. 10). They are powered by means of a capacitor bank, discharged after quench detection. The heat is transferred through thin polyimide insulation layer into the coil and provokes quench in large volume of magnet coil. The heater strips are partially plated with copper to reduce their resistance. The heaters provoke a quench in the cable only below a non-plated part and the natural quench propagation drives the entire conductor into the normal conducting state. In the steady state quench test case, one quench heater strips circuit is powered in DC mode, leaving the rest of quench heaters in magnet protection mode. It allows provoking quenches in function of the power dissipated in the selected quench heater (see Fig. 11).

As an example, the numerical simulations and measurement results obtained on the MQM magnets are shown in Fig. 11. The difference between measured and simulated values is shown in Fig. 12. For all other investigated magnet the largest difference between measurements and simulations was found to be of the order of $20 \%$, whereas the majority of the differences did not exceed $10 \%$.

The first results of the simulation with typical Gaussian beam loss profile show that the quench level for MQM magnets family reads $6 \mathrm{~mW} / \mathrm{cm}^{3}$ and $4 \mathrm{~mW} / \mathrm{cm}^{3}$ for nominal and ultimate currents respectively. The corresponding values for MQY magnets are $8 \mathrm{~mW} / \mathrm{cm}^{3}$ and $5 \mathrm{~mW} / \mathrm{cm}^{3}$.

${ }^{1}$ This value could vary according to the quench heater precision specifications. This implies differences of measured quench heaters resistance for each magnet.

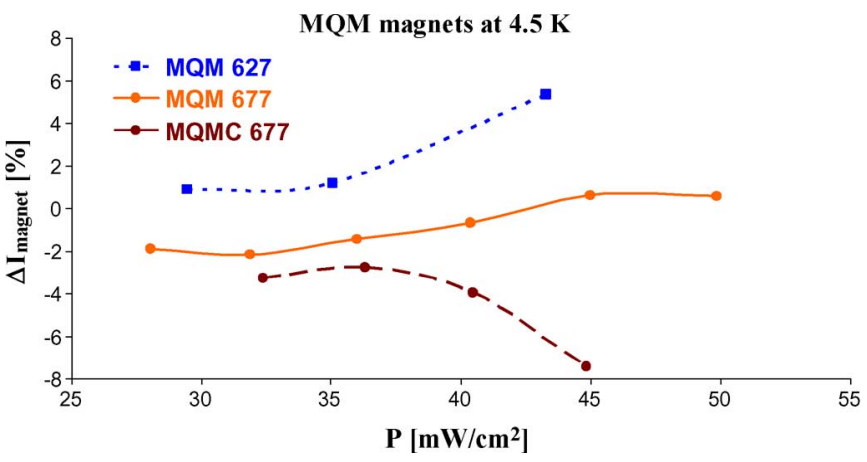

Fig. 12. The relative difference between measurements and simulations for MQM magnet family. The power dissipated in the quench heaters is shown on $\mathrm{x}$ axis and relative difference between measurements and simulations is depicted on y-axis.

\section{CONCLUSION}

The knowledge of the quench levels will allow setting appropriate initial threshold values for the beam loss monitor system. It is expected to increase the operational efficiency of the LHC with the accurate knowledge of the quench levels. The performed measurements and simulations show a maximal relative difference of $20 \%$. This precision is regarded as sufficient for the quench level knowledge. The developed Network Model will be used in the future for the quench limit calculation of other LHC magnet types working at $4.5 \mathrm{~K}$ as well as $1.9 \mathrm{~K}$.

\section{ACKNOWLEDGMENT}

The authors thank J. Bielski, A. Bonasia, M. Calvi, G. D'Angelo, J. Kaplon, L. Oberli, R. Ostojic, P. Pugnat, and $\mathrm{D}$. Tommasini for their help and useful discussions.

\section{REFERENCES}

[1] LHC Design Report, CERN-2004-003, vol. 1.

[2] R. Schmidt et al., "Beam loss scenarios and strategies for machine protection at the LHC," CERN, LHC Project Report 665, 2003.

[3] J. B. Jeanneret, "Measurement of the beam losses in the LHC rings," CERN, LHC-BLM-ES-0001.00, 2003.

[4] F. Bordry et al., "Machine protection for the LHC: Architecture of the beam and powering interlock systems," LHC Project Report 521, 2001.

[5] D. Bocian, G. D'Angelo, and A. Siemko, "The measurements dedicated to the quench limit calculation in the main LHC superconducting magnets at $4.5 \mathrm{~K}$," LHC Project Note, in preparation.

[6] D. Bocian, J. Halik, and A. Siemko, "The measurements dedicated to the quench limit calculation in the main LHC superconducting magnets with an internal heating apparatus," LHC Project Note, in preparation.

[7] P. P. Granieri et al., "Stability analysis of the LHC cables for transient heat depositions," presented at this conference, MT20-1N09.

[8] D. Bocian, B. Dehning, and A. Siemko, "Steady-state beam loss studies in the LHC superconducting magnets," LHC Project Note, in preparation.

[9] A. Verweij, private communication.

[10] Cryosoft, France, 2001.

[11] S. W. Van Sciver, Helium Cryogenics. New York: Plenum Press, 1986.

[12] C. Schmidt, Review of Steady State and Transient Heat Transfer in Pool Boiling Helium I. Saclay, France: International Institute of Refrigeration: Commision A1/2-Saclay, 1981, pp. 17-31.

[13] M. Nishi et al., "Boiling helium heat transfer characteristics in narrow cooling channel," IEEE Trans. Magn., vol. 19, no. 3, pp. 390-393, 1983.

[14] J. B. Jeanneret et al., CERN, LHC Project Report 44, 1996. 